\title{
BESPRECHUNGSAUFSATZ
}

\section{Gerichte, Recht und Verfassungen Lateinamerikas in der neueren Literatur}

Von Wolfgang Spoerr

I.

Yash Ghai / Robin Luckham / Francis Snyder (eds.)

The Political Economy of Law - A Third World Reader

Oxford University Press, Delhi / Bombay / Calcutta / Madras, $£ 32.50$

Der von in Großbritannien lehrenden Universitätslehrem des Rechts und der Soziologie herausgegebene Band "The Political Economy of Law - A Third World Reader" beeindruckt durch die thematische Breite des Materials, das einbezogen wurde. Zusammengetragen aus ganz unterschiedlichen, weit verstreuten Quellen, informieren die Beiträge etwa über "Arbeitsrecht und Arbeitsverhältnisse in Chile" (Ietswaart, ein Beitrag aus dieser Zeitschrift), "Indische Supreme Court-Richter - ein Porträt" (Gadbois), "Die Rolle der Gerichte im ländlichen Mexiko" (Hurt/Hurt) und "Anden-Frauen unter spanischer (Kolonial-) Herrschaft". Die Perspektive ist nicht eine spezifisch lateinamerikanische, sondern die der gebietsübergreifenden Drittwelt-Forschung.

Das Buch ist in acht Abschnitte untergliedert: Der erste untersucht die Entstehung des bürgerlichen Rechtsstaats in Europa und die Erfahrungen mit seiner Übertragung auf die Kolonien. Besonders interessant ist hier der Beitrag von Seidmann über die Rezeption des englischen Rechts im kolonialen Afrika. Der zweite Abschnitt befaßt sich mit "Staat, Recht und Ideologie", der daran anschließende Abschnitt nimmt "Recht und die Internationalisierung des Kapitals" in den Blick. Die Beiträge dieses Teils werden dem Thema weniger gerecht als die der anderen Abschnitte. "Staat, Recht und Wirtschaft", "Der Justizapparat", "Der Juristenberuf und die Klassenstruktur" hingegen machen interessante, empirische Fallstudien zugänglich. Für Juristen zugleich faszinierend und beunruhigend ist die Studie von Toharía über die (spanische) Koexistenz von unabhängiger Justiz und autoritärem Regime. 
Der letzte Abschnitt über "Recht und soziale Transformation" ist derjenige, der einen pragmatisch denkenden Juristen am meisten interessiert. Das wiedergegebene Material aus Chile 1970-73, auf das sich das Kapitel im wesentlichen beschränkt, ist gerade deshalb interessant, weil es Primärliteratur (einen Briefwechsel zwischen dem Obersten Gericht Chiles und Präsident Salvador Allende) ausführlich wiedergibt. Dieser Teil ist überaus interessant zu lesen, er zeigt aber auch, welcher Art sozialer Transformation die Autoren im Auge haben. Wenn die Herausgeber die Dominanz marxistischer Positionen damit begründen, einen bürgerlich-liberalen "Bias" der Juristen ausgleichen zu wollen (Einf. S. 8), so erscheint fragwürdig, ob diese Prämisse auch innerhalb ihres Faches und vor allem in der Dritten Welt zutrifft, für die das Buch vor allem bestimmt ist (Einf. S. 6). Daß die angebotenen Konzepte für die Überwindung der elementaren Probleme der Dritten Welt nützlich sind, bezweifelt der Rezensent; der Nutzen dieses Buches für die wissenschaftliche Diskussion hingegen ist unbestritten. Er wäre aber noch höher gewesen, wenn wenigstens die Fußnoten und Literaturnachweise der Originalbeiträge, zumindest teilweise, erhalten geblieben wären. $\mathrm{Da}$ die fast 90 Beiträge teilweise erheblich gekürzt wurden, ist sicher unvermeidlich. Beiträge marxistischer Klassiker (Marx und Engels) als auf wenige Seiten gekürzte Versatzstücke abzudrucken, ist jedoch eher fragwürdig. Auch wenn man berücksichtigt, daß sich das Buch seiner Anlage nach vor allem an "undergraduates" wendet, wäre ein weniger versatzstückhaftes Vorgehen den referierten Theorien sicher angemessener gewesen.

Alles in allem: Insbesondere durch seine thematische Breite ist der Band als Beitrag zur Diskussion über Recht in der Dritten Welt einzigartig. Er ermöglicht einen recht umfassenden Überblick über Literaturbeiträge, die ansonsten teilweise nur schwer zugänglich sind.

\section{II.}

\section{Krystian Complak}

Los Gobiernos de facto en America Latina 1930 - 1980

Aus dem Polnischen ins Spanische Übersetzt von Alberto Amengual

Biblioteca de la Academia Nacional de la Historia, Caracas 1989

Staatsstreichregierungen - grob gesagt solche, die auf nicht verfassungsmäßigem Wege an die Macht gekommen sind - haben die politische Wirklichkeit Lateinamerikas in großem Umfange geprägt. Sie sind ein genuin gesamt-lateinamerikanisches Phänomen. Das Buch von Krystian Complak ist eine breit angelegte, vergleichende Studie über Installation der Staatsstreichregierungen, ihren institutionellen Aufbau, über Policies und institutionelle Formen der Transition zurück zur Demokratie. Die Methode ist "historisch-deskriptiv" (S. 12). Deshalb ist das einleitende Kapitel über die Ursachen mangelnder politischer Stabilität in Lateinamerika am wenigsten überzeugend, insbesondere der erste Teil über Wider- 
sprüche in Wirtschafts- und Sozialstruktur. Hier argumentiert der Verfasser ganz auf der Basis der im Ostblock üblichen Erklärungsmuster, die mit manchen Spielarten der Dependenztheorie (naturgemäß) Parallelen aufweisen.

Das Bemerkenswerte ist, daß diese einleitenden Beschreibungen von Kausalzusammenhängen inhaltlich (so z.B. mit der fragwürdigen Beschreibung des Autoritarismus als Folge vertiefter Industrialisierung; S. 24) die Analyse im übrigen und ihren Wert in keiner Weise beeinträchtigen. Im Gegenteil: Was folgt, ist die wohl gründlichste uns vorliegende vergleichende und umfassende Analyse des Phänomens; kenntnisreich, ohne ausschweifend zu sein, stets mit Einbeziehung von schwierig zu beschaffendem lateinamerikanischen Primärmaterial. Trotz des Anspruchs, nur historisch-deskriptiv zu arbeiten, treten immer wieder interessante Schlußfolgerungen zu Tage. Einleuchtend die Begründung der Stärke des Militärs trotz zahlenmäßig und finanziell niedriger Ausstattung. Ähnlich die Beschreibung der Ideologie der Putschisten, die regelmäßig sowohl sozialistische wie kapitalistische Inhalte ablehnten.

Die politisch-institutionelle Sichtweise bringt es mit sich, daß auch und gerade auf die Stellung der Gerichte der Blick gerichtet wird (S. 58 ff., 85 ff.). Dabei wird geschildert, wie die Formen weitgehend intakt bleiben und nur in Randbereichen angetastet werden müssen, um zu ganz anderen Inhalten zu führen (S. $78 \mathrm{ff} ., 87 \mathrm{ff}$.).

Es ist eine unbedingte Bereicherung der Literaturlage, daß die Arbeit des Breslauer Hochschullehrers über den Umweg einer venezolanischen Übersetzung ins Spanische "allgemein zugänglich" gemacht wurde.

III.

Hans-Uwe Erichsen (Hrsg.)

Lateinamerika und Europa im Dialog

Menschenrechte - Wirtschaftliche Verflechtung - Menschenbild, Minderheiten, Medien Politische Beziehungen

Duncker \& Humblot, Berlin 1989, 332 S., DM 34,--

Lateinamerika und Europa im Dialog - Programm einer Vortragsreihe der Universität Münster zu den Themen Menschenrechte, Wirtschaftsbeziehungen, Medien und politische Beziehungen. "Zur philosophischen Begründung der Menschenrechte" bzw. zu "Pflichten und Rechte der Menschen - ein elementarer Tausch" referieren Reinhardt Brandt und Ottfried Höffe. Ihre Beiträge sind spezifisch lateinamerikanisch nur deshalb, weil auf dem Subkontinent das Thema Menschenrechte eine große Rolle spielt. 
Die Verschuldungskrise wird in zwei unterschiedliche Positionen einführenden Referaten behandelt. Der Beitrag von Cesar Maldonaldo über "Selbsthilfe und ländliche Entwicklung in Lateinamerika" ist der einzige, der auf empirischem Material beruhend sozialwissenschaftlich deduziert und (eingehende) Literaturnachweise enthält. Wenn auch nicht wissenschaftlich aufgebaut, so beeindruckt der Beitrag von Walter Haubrich über "Lateinamerika in europäischen Medien" doch durch seine Ausgewogenheit und seinen Kenntnisreichtum. Den Europäern wird der Spiegel ihrer Vorurteile über Lateinamerika vor Augen gehalten.

Schade, daß über den "workshop" "Verfassung und politische Herrschaftsstrukturen in vergleichender Perspektive" inhaltlich (im Gegensatz zu den anderen Workshops) nichts mitgeteilt wird; dabei weckt die Liste der Referate hohe Erwartungen. Eine politisch bekannte Debatte wird auch in diesem Sammelband ausgetragen zwischen Leonardo Boff, brasilianischer Bischof und Vertreter der Befreiungstheologie, und Martin Kriele, deutscher Jurist und scharfer Kritiker der Theologie der Befreiung. Es ist dies eine Debatte, die Politikwissenschaftler, Theologen und Juristen gleichermaßen angeht und interessiert.

Insgesamt liefert der Band interessante Diskussionsbeiträge; eine wissenschaftliche Einführung in die Themen des Titels darf man sich jedoch nicht versprechen.

IV.

Hans-Rudolf Horn / Albrecht Weber (Hrsg.)

Richterliche Verfassungskontrolle in Lateinamerika, Spanien und Portugal

Nomos Verlagsgesellschaft, Baden-Baden 1989, 220 S., DM 68,--

Richterliche Verfassungskontrolle, d.h. die Befugnis, Akte der gesetzgebenden, exekutiven und judikativen Gewalt auf ihre Vereinbarkeit mit der Verfassung zu überprüfen, ist ein überaus reizvolles Thema der Verfassungsvergleichung. Der von Horn und Weber herausgegebene Band ist ein großer Schritt nach vorn in der Forschung auf diesem Gebiet.

Von den fünf Beiträgen befassen sich zwei mit Spanien ("Der richterliche Schutz der Grundrechte in Spanien" von Karl Peter Sommermann und "Richterliche Normenkontrolle in Spanien" von Albrecht Weber). Spanien ist ein Thema, das monographisch schon sehr gut aufgearbeitet ist, so daß die beiden Beiträge hier vor allem als komprimierte Einführung wertvoll sind. Neuland für die deutsche Literatur betritt hingegen Jorge Mirada mit seinem Beitrag über "Die verfassungsgerichtliche Kontrolle in Portugal"). Einige Beispiele aus der Rechtsprechung und damit dem Verfassungsleben hätte man sich aber zur Untermauerung schon gewünscht. 
Im zweiten Teil wird Lateinamerika in die Betrachtung einbezogen; "Politische und soziale Grundrechte in Lateinamerika" (Hans-Rudolf Horn) und "Die Verfassungskontrolle in Lateinamerika" (Hector Fix-Zamudio) sind die Themenstellungen. Besonders der letzte Beitrag ist eine breit angelegte, alle iberoamerikanischen Verfassungen einbeziehende gründliche vergleichende Darstellung. Trotz der recht tiefgehenden Einführung auch in technische Einzelheiten kommt die vergleichende und systematische Perspektive nie zu kurz.

Es ist mehr und mehr üblich geworden, Spaniens Verfassungssystem im europäischen Kontext, losgelöst vom iberoamerikanischen Zusammenhang zu sehen. Intensive innerwesteuropäische Rezeptionsprozesse rechtfertigen dies. Der vorliegende Band ist gewissermaßen eine "Gegenbewegung", indem er die spanisch-iberoamerikanische Perspektive einbezieht. $\mathrm{Da} \beta$ es dafür nicht nur sprachliche, sondern auch historische und systematische Gründe gibt, wird an mehreren Stellen deutlich. Sowohl die unterschiedliche Komplexität der prozessualen Systeme wie auch die grundverschiedene reale Wirkungskraft der Verfassungsgerichtsbarkeit, wie sie in den Beiträgen zu Tage treten, zeigen aber auch, daß Spanien und Lateinamerika in den letzten 15 Jahren einen sehr unterschiedlichen Weg gegangen sind. 